Matgeraidad del Haspizal

Batros Luco

Prof, González C.

\title{
ALgo MAS SOBRE PREMATURIDAD
}

Por el Dr. ROBERTO KOHAN I.

Asesor Pzdiátrico de la Maternidad del Hospital R. Barros Luco.

Hace algún tiempo el Dr. Illanes y uno de sus ayudantes bicieron una brillante exposición sobre la prematuridad. Pues bien. rosotros también queremos decir algunas palabras a este respecto, pero precisando, desde ya, que nuestro trabajo no viene a ser una crítica a los autores ya citados, sino un agregado, un grano de arena más, al mejor conocimiento de la prematuridad, tal como se presenta entre nosotros.

Nada hay más aburridor que un trabajo de estadística. pero tampoco nada precisa más los hechos que un estudio estadístico, hecho a conciencia y sin ánimo preconcebido. Es decir, hemos tratado de poner en práctica la máxima de Buffon: "Reunamos hechos para tener ideas".

Nuestra investigación se basa en la observación de 2,815 niños nacidos en la Maternidad del Hospital Barros Luco, desde el $10^{\circ}$ de enero de 1938 al 31 de diciembre de 1939.

El total de niños con un peso inferior a 2,500 grs. fué de 345 . lo que da un porcentaje de $12.29 \%$. proporción sensiblemente igual a la observada por la mayoría de los autores extranjeros. ¿Cómo se explica, entonces, que en una de las últimas sesiones de la Sociedad de Pediatria, hayamos afirmado que el porcentaje de prematuridad alcanzaba a un $25-30 \%$ ?

En Obstetricia existe el concepto que es prematuro el niño que nace antes del término normal del embarazo, concepto que, en justicia, refleja la realidad de los bechos. Pero, en Pediatría, se piensa que es prematuro el niño que nace con un peso inferior a 2,500 grs. 
El número de niños nacidos al octavo y medio meses de la vida intrauterina, fué de 406 que, sumados a los 345 niños nacidos con un peso inferior a 2.500 grs. da un porcentaje de $26.6 \%$.

Ahora bien, con el objeto de evitar toda posibilidad de confusión, nos referiremos, únicamente, a los 345 niños nacidos en nuestra Maternidad, con un peso inferior a 2.500 grs.

Como es sabido, la clasificación de la Liga de las Naciones, respecto al recién nacido. es la siguiente:

Niño a términa: Más de 2.500 grrs., $47.1 \mathrm{~cm}$. . 38 semanas de vida intrauterina.

Prematuro previable: 400 a 999 grs., 28 a $35 \mathrm{~cm}$. de largo, de 22 al final de 28 semanas.

Prematuro viable: 1.000 a 2,499 grs., 35.1 a $47 \mathrm{~cm}$., 29 al final de 37 semanas.

Nosotros tenemos en nuestra estadistica:

Prematuros previables

Prematuros viables.

Para la determinación del peso, hemos seguido la clasificación universalmente aceptada, de Ylppo, que es la que sigue:

1. Niños que pesan de 600 a 1,000 grs.

2. Niños que pesan de 1,001 a 1,500 grs.

3. Niños que pesan de 1,501 a 2,000 grs.

4. Niños que pesan de 2,001 a 2,500 grs.

Entre nosotros, la proporción es la siguiente:

$\begin{array}{cccc}600-1.0000 & 1.001-1,500 & 1,501-2.000 & 2.001-2,500 \mathrm{grs} \\ 5 & 49 & 9 \mathrm{I} & 200\end{array}$

El mayor peso de los prematuros oscilaba entre 2,001 y 2,500 grs., lo que da un $57,9 \%$. El peso más bajo lo encontramos en un niño de 640 grs., que alcanzó a vivir 12 horas.

Es un hecho comúnmente observado, que todo reciénnacido baja de peso en los primeros dias de su vida. Sin embargo, un estudio hecho por nosotros a este respecto nos hace ver que la baja $\mathrm{d}_{e}$ peso, en los niños que alcanzaron a pasar las primeras 24 horas críticas, fué sólo de un $66,2 \%$. 
Oscilaciones en el peso.- Se determinó en 243 casos.

\begin{tabular}{|c|c|c|}
\hline $\begin{array}{l}\text { descenso } \\
61 \quad(66,2 \%)\end{array}$ & igual & $\begin{array}{l}\text { ascenso } \\
(22.2 \%)\end{array}$ \\
\hline
\end{tabular}

Es verdad que hay acuerdo en hablar de prematuros previables y prematuros viables, pero nosotros hemos querido determinar en qué época del embarazo es más frecuente la prematuridad y hemos obtenido los siguientes datos:

$5 \mathrm{~m} .6 \mathrm{~m} .61 / 2 \mathrm{~m} . \quad 7 \mathrm{~m} . \quad 7 \mathrm{y} / 2 \mathrm{~m} . \quad 8 \mathrm{~m}$. partos gemelares $\begin{array}{lllllll}2 & 31 & 32 & 66 & 52 & 126 & 18(36)\end{array}$

o sea, que el mayor porcentaje se presenta a los 8 meses, con un $36 \%$.

Sexo. -- Woodbury encontró una predominancia de los varones.

Nosotros:

$$
\begin{aligned}
& \text { sexo femenino: } 176(51 \%) \text {. } \\
& \text { sexo masculino: } 169(49 \%) \text {. }
\end{aligned}
$$

Es decir, la prematuridad se presenta, prácticamente, en igual proporción entre los hombres y las mujeres.

Talla del prematuro: Se determinó en 254 casos.

$28-35 \mathrm{cms}$.

35-40 cms.

$40-45$ cms.

100

$45-47 \mathrm{cms}$.

139

Porcentaje mayor, de 45 a 47 cms., en un 54.7\%\%.

Caracteres del prematuro de ocho meses. - El estudio de los prematuros nacidos al octavo mes de la vida intrauterina, nos da los siguientes detalles:

Peso

Largo

Circunferencia craneana

Circunferencia toráxica

Circunferencia abdominal

Coeficiente de vitalidad
2,400 grs.

$46 \mathrm{cms}$. (oscila entre 45 y $47 \mathrm{cms}$.)

$32 \mathrm{cms}$. (oscila entre 31 y $32 \mathrm{cms}$.)

$29 \mathrm{cms}$. (oscila entre 28 y $30 \mathrm{cms}$.)

$27 \mathrm{cms}$. (oscila entre 27 y $28 \mathrm{cms}$.)

Oscila entre 1.4 y 1.5 . 
Presentación del prematuro: Se considera que la posición anormal del feto favorece el parto prematuro. Nosotros tenemos los siguientes datos al respecto:

\begin{tabular}{ccccc}
\hline cefálica & nalgas & tronico & pattos gemelares & $\begin{array}{c}\text { nacidos fuera } \\
\text { del servicio }\end{array}$ \\
\hline $242(70 \%)$ & 39 & 13 & $18(36)$ & 15
\end{tabular}

Este dato es interesante, porque si consideramos todos los niños recién nacidos, comprobaremos que el niño se encuentra en posición de vértice. por lo menos, en un $85 \%$. Hay que pensar, entonces, que alguna acción debe ejercer la defectuosa posición del niño.

Maniobras obstétricas: En relación con la presentación del feto, debemos considerar las maniobras obstétricas.

Tenemos 17 casos, lo que da un $4.7 \%$, clasificados en la siguiente forma:

Forceps: Dos casos (uno muerto).

Versión interna: 9 casos.

En tres ocasiones, se hizo con feto muerto en el útero.

Tres niños fallecieton luego después de la intervención.

Todos estos datos nos hacen ver que las maniobras obstétricas influyen más en la mortalidad del prematuro que en su producción.

La madre del prematuro: Ahora nos referiremos a los caracteres de la madre que da a luz el niño prematuto.

Edad de la miadre.- Se determinó en 327 casos.

1938

$\begin{array}{cccc}15-20 & 20-25 & 25-30 & 30 \text { años ó más } \\ 33 & 46 & 24 & 40 \\ 34 & 65 & 41 & 44 . \\ 67 & 111 & 65 & \frac{84}{21}\end{array}$

Mayor porcentaje, entre $\operatorname{los} 20$ y $\operatorname{los} 24$ años (inclusive) : $33.9 \%$. forma:

Estas madres se encuentran clasificadas en la siguiente

\begin{tabular}{cccc}
\hline Primiparas & $\begin{array}{c}\text { Ptimíparas } \\
\text { (con antecedentes } \\
\text { de abortos) }\end{array}$ & Secundipatas & Moltipa tzs \\
\hline 127 \\
$140(42.9 \%)^{13}$ & 60 & $187(57.1 \%)^{127}$
\end{tabular}


Antecedentes de partos prematuros: 29 casos $(8.8 \%$ ).

Madres con antecedentes de abortos habitualiz: 44 casos

\section{$(13.4 \%)$}

Primíparas de 30 años ó más: 5 (4 casadas, 1 soltera), lo que da un $5.9 \%$.

No olvidemos que el número de madres de 30 años ó más, fué de 84 , es decir, un $25.6 \%$.

Como detalles agregados, anotemos que el mayor número de hijos lo ha tenido una señora de 34 años. Tenía los siguientes antecedentes: 15 hijos. 2 abortos espontáneos, 4 hijos vivos. Reacción de Wassermann (-). Da a luz un niño de 1,100 grs, muerto en el útero.

Fijémonos en la gran cantidad de hijos que ha tenido esta mujer. pero observemos que sólo 4 de estos niños están vivos, fenómeno muy común entre las mujeres del pueblo chileno.

Por otra parte, la mujer con más hijos vivos, tiene estos antecedentes: 36 años, casada, trabaja en los quehaceres de la casa. 11 hijos, 9 vivos, nace un niño de 2,080 gits. en regulares condiciones.

Ahora, relacionemos estos hechos con lo observado por algunos autores extranjeros.

Dunharn y Mc Âlennzy diren que el número de primiparas es igual al de las multíparás. mientras que nosottos encontramos un porcentaje un poco mayor de multiparas.

Murphy encontró 12 en una serie de 44 madres multiparas que habian tenido previamente partos prematuros, mientras que nosotros sólo evidenciamos un $8,8 \%$ de prematuridad habitual.

En seguida. para Capper, los abortos habituales se presentan en un $2.8 \%$.

Según Ylppö, en un $0.6 \%$. Nosotros tenemos madtes con antecedentes de abortos habituales en un $13.4 \%$.

Ress demuestra que la prematuridad es más frecuente en los meses fríos, en lo cual estamos absolutamente de acuerdo, ya que el mayor número de prematuros lo observamos en pleno invierno.

Lof quist, de Suecia, dice que los casos de prematuridad se ven, con mayor frecuencia, en los niños de las madres primiparas, especialmente si ellas son de edad avanzada. Pero, nuestras estadisticas nos dicen que en an total de 84 madres de 30 años o más, sólo cinco eran primíparas, porcentaje que habla por sí solo. 
La nutrición de la madre tiene una importancia fundamental en la prematuridad. Por ejemplo. Schneider comprobó que el número de prematuros aumentó durante la Guerra Mundial. Entre nosotros, basta echar un vistazo a cualquiera de las Salas de la Maternidad para afirmar categóricamente que la alimentación de la madre tiene un papel decisivo en el desarrollo de la prematuridad. Lo único que nos debe sorprender es que cómo es posible que sea tan escaso el número de prematuros en nuestro medio hospitalario.

Condición social de la madre:

\begin{tabular}{|c|c|c|c|}
\hline & casadas & solteras & viudas \\
\hline $\begin{array}{l}938 \\
1939\end{array}$ & $\begin{array}{l}74 \\
82\end{array}$ & $\begin{array}{l}67 \\
73\end{array}$ & $\begin{array}{l}1 \\
1\end{array}$ \\
\hline & $156(52.3 \%)$ & $140(46.9 \%)$ & $2(0.8 \%)$ \\
\hline
\end{tabular}

Según Crawford, la ilegitimidad se presenta en un $17.3 \%$. Jazger encuentra un $36.5 \%$. Nosotros tenemos el record, $46.9 \%$.

Picciali hizo un estudio de 3.341 recién-nacidos, de los cuales 802 eran ilegítimos. En estos niños, el peso y la talla fueron inferiores a los de los niños normales. El peso y la talla fueron superiores en los ilegítimos de las madres multíparas.

La ilegitimidad, en nuestro medio social bajo, tiene que tener alguna importancia en el . desarrollo de la prematuridad, puesto que si tomamos en consideración los 2,815 niños nacidos en los dos últimos años en ninestra Maternidad, veremos que el porcentaje de madres solteras sólo alcanza a un $30 \%$.

Profesión de la madre:

Empleadas

Obreras

Costureras

Lavanderas

Cocineras

Comerciantes

Peinadoras

\begin{tabular}{cc} 
casadas & solt?ras \\
\hline 4 & 27 \\
24 & 26 \\
5 & 5 \\
7 & 5 \\
5 & 6 \\
0 & 5 \\
2 & 0
\end{tabular}

Las madres restantes insistieron en que se dedicaban, exclusivamente, a los quehaceres de la casa. 
Lo primero que resalta es el elevado número de empleadas entre las madres solteras. Las sirvientes constituyen un núcleo de mujeres ideales para tener partos prematuros, por los medios que ponen en práctica para eliminar el producto de la concepción y por los esfuerzos físicos que desarrollan durante el embarazo.

Además, fijémonos en el tremendo trabajo que tienen las mujeres del pueblo en su propia casa y pensemos si ello es o no causa suficiente para que el feto salga del vientre de la madre antes del término normal del embarazo.

Enfermedad de la madre: La revisión de los boletines de las madres con niños prematuros nos ha permitido confeccionat el siguiente cuadro:

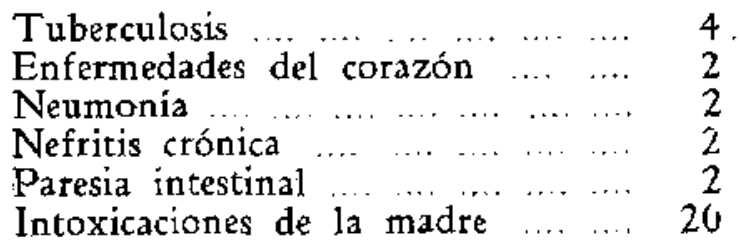

Afecciones locales (genítales) dz l'a madre:

Hidroamnios

Placenta previa

14

Metrorragias abundantes ........... 5

Membranas rotas espontáneamente 26

Madres sifiliticas: $53(16,2 \%)$.
$\mathbb{R}$. Wassermanis +
(f
R. Wassermann ++
26
R. Wassermann + t+ 2.2

Relación feto-placentarja: se determinó en 215 casos.

$\begin{array}{rrrrrrr}1 \times 2 & 1 \times 3 & 1 \times 4 & 1 \times 5 & 1 \times 6 & 1 \times 7 & 1 \times 8 \\ 4 & 23 & 58 & 85 & 34 & 7 & 4\end{array}$

Relación feto-placentaria más común: $1 \times 5$ (39,5\%).

El Dr. Illanes, en su trabajo, comprueba un $47 \%$ de madtes tuberculosas y sifilíticas, porcentaje que vendría a ser el más alto del mundo civilizado. Para corroborar lo que decimos, basta examinar el cuadro siguiente, que se refiere a las 
causas de la prematuridad, según lo observado por autores de distintas nacionalidades.

\begin{tabular}{|c|c|c|c|c|c|c|c|c|c|c|}
\hline & $\begin{array}{l}\text { Ylp } \\
\text { Bre } \\
\text { Pec } \\
\text { Cap }\end{array}$ & $\begin{array}{l}\text { ppi } \\
\text { ese } \\
\text { kar } \\
\text { ppe }\end{array}$ & $\begin{array}{lll} & \ldots & \\
\ldots & \ldots & \ldots \\
\mathbf{n} & \ldots \\
\mathbf{r} & \ldots\end{array}$ & $\begin{array}{ccc}\ldots & \cdots & \\
\ldots & \ldots & \ldots \\
\ldots & \ldots \\
\ldots & \ldots \\
\ldots & \ldots\end{array}$ & $\begin{array}{l}\cdots \\
\cdots \\
\cdots \\
\cdots\end{array}$ & $\begin{array}{r}668 \\
987 \\
4,055 \\
437\end{array}$ & $\begin{array}{l}\text { casos } \\
\text { casos } \\
\text { casos } \\
\text { casos }\end{array}$ & $\begin{array}{l}\text { (año } \\
\text { (año } \\
(a \tilde{n} o \\
(a \tilde{n} o\end{array}$ & $\begin{array}{l}1920) \\
1938) \\
1936) \\
1928)\end{array}$ & \\
\hline & & & $\begin{array}{l}\text { Emb. } \\
\text { mult. }\end{array}$ & Toximia & Sifilis & Corazón & Tbc. & $\begin{array}{l}\text { Plac. } \\
\text { previa }\end{array}$ & $\begin{array}{l}\text { Despzen. } \\
\text { placenta }\end{array}$ & $?$ \\
\hline Ylppo & .. & & 21 & 3.1 & 3.9 & 1,8 & - & - & - & 55.2 \\
\hline Breese & $\ldots$ & & 16.2 & 17,5 & 1.7 & 1 & 0.4 & 4 & 1,6 & 43 \\
\hline Peckiam & n .. & & 11.8 & 16.3 & 16.1 & 2.1 & - & 2.5 & 2 & 27.9 \\
\hline Capper & r ... & .. & 15.1 & 1.4 & 3 & - & 5.5 & 一 & - & 60,2 \\
\hline
\end{tabular}

El porcentaje más alto para la tuberculosis de la madre fué de un $5.5 \%$ y, para la sífilis, de $16.1 \%$. En nuestro Hospital, los porcentajes fueron de 1,2 y $16,2 \%$. Seyuramente, el porcentaje para las madres tuberculosas es un poco más elevado, ya que algunos casos deben haber pasado desapercibidos, por tratarse de enfermas con lesiones muy discretas. En todo caso, no puede pasar de un 3 a un $4 \%$. El gran número de prematuros, hijos de madres tuberculosas, comprobado por el Dr. Illanes, se debe, como él ya lo ha explicado, a que en la Casa Nacional del Niño se reciben muchos niños con antecedentes tubetculosos.

Todos los autores están de acuerdo en conceder importancia fundamental a los embarazos múltiples, a las toxemias $y$ a las infecciones crónicas.

Tampoco hay que olvidarse de los partos gemelares (en nuestra estadística, 18) como causantes de niños con un peso inferior a $2.500 \mathrm{grs}$. No cabe duda que las afecciones locales de la madre también influyen en un mayor porcentaje de prematuridad.

Prematuros fallecidos: $113(32,7 \%)$.

Siguiendo la clasificación de Ylppö, los resultados son. los siguientes: 
$600-1,000 \quad 1,001-1,500 \quad 1,501-2,000$

$2.001-2,500$

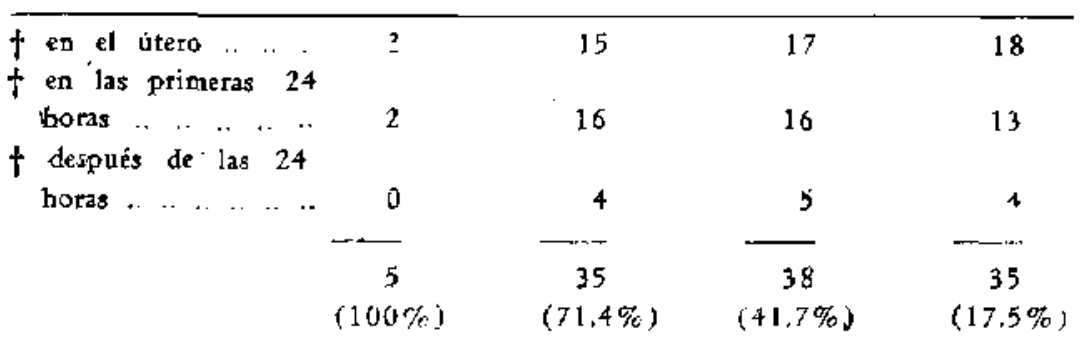

Muertos en el útero

Muertos antes de las 24 horas

$53(46,9 \%)$

Muertos después de las 24 horas

A primera vista, el porcentaje de mortalidad es bastan. te elevado, pero si sólo tomamos en cuenta los niños prema. turos que nacen vivos, vemos que este porcentaje desciende bruscamente y entra dentro de los límices observados por la mayoria de los autores extranjeros. Desde luego, en los niños con un peso inferior a 1.000 grs., la mortalidad ha sido de un $100 \%$; en los prematuros de 1,001 a 1,500 grs., de un $71.4 \%$. De los 1.501 a $\operatorname{los} 2.000$ grs. de un $41.7 \%$. Finalmente, en los niños de 2,001 a 2.500 grs., de $17,5 \%$.

Un punto importante sería determinar las causas de mortalidad en estos prematuros. Desgraciadamente, no podemos anotarlas, por no haberse hecho la autopsia en todos estos niños.

Dunham y Mc Alenney, en un estudio de 244 prematuros, dan los siguientes detalles:

244 niños: 66 fallecieron.

Primigestas: $194(79 \%)$.

Multiparas: $21 \%$.

Ylppö también comprueba una menor mortalidad en los niños de las madres multíparas, en la siguiente proporción: 16 y $30 \%$.

Nosotros hemos encontrado 39 niños fallecidos, hijos de madres primíparas y 74 hijos de madres multíparas, es decir, b3mos comprobado el siguiente porcentaje: 34.4 y 65.6 por ciento. Ahora bien, en relación a' número total de primigestas y multíparas, tenemos el siguiente porcentaje:

Hijos de madres primiparas Hijos de madres multíparas 
Como se ve. nuestros resultados son totalmente distintos a los obtenidos por los autores extranjeros.

En ciertas publicaciones sobre prematuridad, al considerar su mortalidad, sólo toman en cuenta los niños que nacieron vivos $\mathrm{y}$ que fallecieron más tarde. Si siguiéramos este método, nos encontraríamos con que la mortalidad de los prematuros en nuestro medio hospitalario es únicamente de un $17,3 \%$; de todas maneras estamos lejos de las estadísticas obtenidas por algunos autores europeos y estadounidenses.

Así, Wieland, en el Congreso de Trieste, expuso que hasta el año de 1927, en Basilea, la mortalidad de las prematuros fué de un $17,6 \%$, mientras que, más tarde, descendió a un $9,8 \%$, y la causa estaría en el aislamiento de los prematuros, en la alimentación con leche de mujer y en el tratamiento precoz con vitaminas.

Por otra parte, Lamb. del Brooklyn Hospital, se refie. re a 120 prematuros, de los cuales sólo fallecieron 10 , ocho en las primeras 24 horas. E1 autor anota, orgullosamente, y con justa razón, que estos excelentes resultados se deben al cuidado que reciben los prematuros en dicho Hospital.

\section{Un resumen sobre la materia}

A nuluestro parecer, la mejor clasificación sobre las causas de la prematuridad es la de Hess, publicada en el Prac. tice of Pediatrics.

I. Expulsión de los niños prematuros, debidas: sicos:

a) Lesiones, caídas u otros tipos de agotamientos fí-

b) Alteraciones emocionales bruscas; emocional):

c) Rotura prematura de las membranas (accidental a

d) Procedimientos quirútgicos no obstétricos.

\section{Condiciones que causan algün daño al feto:}

a) Enfermedades constitucionales de los padres;

b) Infecciones crónicas, como la sifilis, la nefritis crónica, la tuberculosis, las enfermedades del corazón y el bocio exoftalmio: 
c) Enfermedades infecciosas agudas;

d) Causas locales, como las enfermedades del endometrio, la infección gonorreica y las posiciones defectuosas del útero;

e) Posiciones anómalas del feto:

f) Embarazos múltiples;

g) Nutrición defectuosa del feto;

h) Intoxicación por el alcohol o metales pesados:

i) Malformaciones congénitas del feto:

j) Edad avanzada de los padres:

k) Influencia de la estación.

Ahora, revisemos nuestro trabajo y podremos comprobat lo siguiente:

1. La presentación del feto tiene alguna importancia. ya que, en los prematuros, la presentación cefálica es menos frecuente que en los niños de término.

2. El sexo no parece influir, puesto que nacen, practicamente, igual número de varones y mujeres.

3. Los partos gemelares dan un porcentaje bastante elevado de niños con un peso inferior a $2.500 \mathrm{gts}$. fríos.

4. La prematuridad es más frecuente en los meses

5. Las madres de 20 a 24 años (inclusive) son las que dan el más alto porcentaje de prematuridad.

6. También interviene la edad avanzada de las ma. dres, ya que encontramos un $25,6 \%$ de mujeres de 30 años o más.

7. La prematuridad es más común entre las multí. paras.

8. La prematuridad habitual se comprueba en un porcentaje muy escaso.

9. La deficiente nutrición del pueblo chileno es una de las causas más importantes de prematuridad.

10. El número de madres solteras en nuestro medio hospitalario es muy marcado, $y$, entre ellas. la prematuridad es frecuente.

11. La tuberculosis, las enfermedades del corazón $y$ otras afecciones de la madre parecen intervenir en un porcentaje reducido.

12. La sífilis desempeña un papel importantisimo en la génesis de la prematuridad. 


\section{Conclusiones}

a) El problema de la prematuridad es más obstétrico que pediátrico.

b) Si llamamos prematuros aquellos niños que han nacido con un peso inferior a $2,500 \mathrm{grs}$., tendremos que la prematuridad se presenta, únicamente, en un $12,20 \%$, en nuestro medio hospitalario.

c) Sin tomar en consideración los prematuros que han nacido muertos, el porcentaje de mortalidad es semejante al obtenido por muchos autores extranjeros.

d) Finalmente, entre nosotros, las causas principales de prematuridad, son:

1. La edad avanzada de la madre:

2. Los partos gemelares;

3. La ilegitimidad;

4. La sífilis; $y$

5. La deficiente nutrición de la madre de la çâse baja.

\section{BIBLIOGRAFIA}

HESS.-Factores etióógicos que infuencian la terminación dé embatazo.

Practice of Pediatries, VI, Cap. 43. Pags. 10 y 11.

LOFQVIS'T"-Klinisch-ststistische Unter uchungen. Uber Frühgeburten. Acta obst. et gynec. Scandinavia. Pg. 161, 1931.

YLPPO.-Zt:chr. f. Kinderb. 1920. Pg. 110.

FINKELLSTEIN.--Parto Prematuro. Tratado de Enfermedades del niño de pe. cho. Pg. 149.1929.

CAPPER.-Th: Fate and Development of the Immature and of the Premature Child. Am. J. Dis. Children. Febreto 1928. Pgi. 262-288.

NINA ANDERSON y $L Y O N$-Causes of Prematurity. Am. J. Dis. Chitdren. Spt. 39, Pg. 536.

DUNHAM y MC ALENNEY,-A Study of 244 prematures. Am. J. Uis. Chi:dren. Dic. 36. Pg. 171 .

CHATTAS.-Oganización moderna de protección al niño prematuro. Arch. Arg. Ped. Spt. 39, Pg. 287.

LAMB.-Factores que influsacian en el porcent?je de mortalidad del nijón prematuro. Brooklyn Hospita'. Abril 39, Pg. 69

WHELAND.-Jos niños prématuros en la Clínica Infantil de Basilea. Congreso de Trieste.

PICClOLI.—Datos estadisticos sobre el recién-natide ilegítimo. Il Lattante. Encro 39, Py. 3.

BRANDER.-Cau as del parto prematuro. Finsk-laek-sáellsk-Handl. Pg. 1010. Oct. 38 .

KEIZER.-Causas of Premature Bitth. Am. J. Dis. Children. Enero 40. Pg. 169. 